\title{
Pseudogenization of trnT-GGU in chloroplast genomes of the plant family Asteraceae
}

\author{
Abdullah $^{1, *}$, Furrukh Mehmood ${ }^{1}$, Parviz Heidari $^{2}$, Ibrar Ahmed ${ }^{*}, 3$, Peter Poczai ${ }^{*, 4,5,6}$ \\ ${ }^{1}$ Department of Biochemistry, Faculty of Biological Sciences, Quaid-i-Azam University, \\ 45320 Islamabad, Pakistan \\ ${ }^{2}$ Faculty of Agriculture, Shahrood University of Technology, 3619995161 Shahrood, Iran \\ ${ }^{3}$ Alpha Genomics Private Limited, 45710 Islamabad, Pakistan \\ ${ }^{4}$ Finnish Museum of Natural History, P.O. Box 7, FI-00014 University of Helsinki, Finland \\ ${ }^{5}$ Faculty of Biological and Environmental Sciences, PO Box 65 FI-00065 University of \\ Helsinki, Finland \\ ${ }^{6}$ Institute of Advanced Studies Köszeg (iASK), PO Box 4, H-9731 Köszeg, Hungary \\ * Correspondence: Abdullah (abd.ullah@bs.qau.edu.pk) \\ IbrarAhmed (iaqureshi_qau@yahoo.com) \\ Péter Poczai (peter.poczai@helsinki.fi)
}




\begin{abstract}
The chloroplast genome evolves through the course of evolution. Various types of mutational events are found within the chloroplast genome, including insertions-deletions (InDels), substitutions, inversions, gene rearrangement, and pseudogenization of genes. The pseudogenization of the trnT-GGU gene was previously reported in the Cryptomeria japonica (Cupressaceae), Pelargonium x hortorum (Geraniaceae), and in the two species of the tribe Gnaphalieae (Asteroideae, Asteraceae). Here, we performed a broad analysis of the trnT-GGU gene among the species of twelve subfamilies of Asteraceae and found pseudogenization of this gene is not limited to the two species of Gnaphalieae or the tribe Gnaphalieae. We report for the first time that this gene is pseudo in the species of three subfamilies of Asteraceae, including Gymnarrhenoideae, Cichorioideae and Asteroideae. The analyses of the species of 78 genera of Asteroideae revealed that this pseudogenization event is linked to the insertion within the $5^{\prime}$ acceptor stem and not linked to the habit, habitat, and geographical distribution of the plant.
\end{abstract}

\title{
Key words
}

Chloroplast, Asteraceae, Asteroideae, trnT-GGU, Pseudogenization, insertions-deletions (InDels) 


\section{Introduction}

The plant family Asteraceae (Compositae), commonly known as the daisy or sunflower family, is among the three megadiverse families which comprise up to $25 \%$ of angiosperm species [1]. Asteraceae comprises up to $10 \%$ species of all the flowering plant with 25,000 35,000 estimated species, is comparable only to Fabaceae and Orchidaceae [1]. These species are diverse in distributions and habitat, exist on every continent, including Antarctica, and occupied every type of habitat [1,2]. This family is divided into 13 subfamilies $[1,3,4]$. The subfamily Asteroideae is the youngest and largest subfamily of Asteraceae, comprising $17000+$ species $[1,4]$.

The chloroplast is the vital organelle in plants due to its role in photosynthesis [5]. This is prokaryotic by the origin and shows uniparental inheritance paternal in some gymnosperm and maternal in most angiosperms [6-8]. The uniparental inheritance and differential mutation rate of different regions of the chloroplast genome makes it suitable for studies ranged from population genetics to phylogenetics $[9,10]$. Many mutational events occurred with chloroplast genomes, including InDels (insertions-deletions), substitutions, inversions, copy number variations, etc [11-18]. These various types of mutational events also lead to complete deletion or pseudogenization of the functional genes within the chloroplast genome, including protein-coding genes, and transfer RNA genes [19-21].

Two transfer RNA genes exist for threonine, which lies in the large single-copy region of the chloroplast. One copy of threonine (trnT-GGT) lies between atpA and psbD along with two other transfer RNA genes, including trnG-UCC and trnR-UCU. Another copy of threonine exists in tnT-F regions, which are widely used for phylogenetic analyses $[4,22]$. The pseudogenation of trnF-GGA has been reported in some plant lineages [20,23,24]. Previously the pseudogenization of trnT-GGT has been reported based on comparative genomics of the two species of tribe Gnaphalieae (Asteroideae, Asteraceae). However, the authors suggested that this pseudogenization of trnT-GGT will be limited to the tribe Gnaphalieae [25]. In the current study, we analyzed the trnT-GGT genes throughout the family Asteraceae to get the data about the extent of pseudogenization. We report that $\operatorname{trnT}$-GGT is pseudogene/deleted in all the species of the subfamily Asteroideae.

\section{Materials and methods}

The complete chloroplast genome sequences of 125 species were retrieved from the National Center for Biotechnology and Information (NCBI) belonging to six subfamilies of Asteraceae 
(Table S1). These included chloroplast genome sequences of 97 species of Asteroideae, 13 species of Cichorioideae, 11 species of Carduoideae, 2 species of Barnadesioideae, and one species each of Mutisioideae, Stifftioideae and Pertyoideae. The raw reads of 10 other species were retrieved from Sequence Read Archive (SRA) to extract trnT-GGU gene. This helped us to include the data of seven other subfamilies, including Gymnarrhenoideae, Corymbioideae, Famatinanthoideae, Gochnatioideae, Hecastocleidoideae, and Wunderlichioideae, Stifftioideae (Table S2). The raw read of these species was retrieved and mapped to Silybum marianum (KT267161) in Geneious R8.1 [26] using Medium-low Sensitivity/Fast, keeping all other parameters as default. The consensus was annotated and extracted after confirmation of mapping quality, specifically focusing on the trnT-GGT region. We retrieved chloroplast genome sequences of 28 species of Diplostephium, 24 species of Artemisia, and 21 species of Aldama to perform comparative analyses of the $\operatorname{trnT-GGU}$ at genus level in the Asteroideae subfamily. This approach enabled us to include diverse species in our study regarding geographical distribution, habit, and habitat (Table S1). The pseudogenization of trnT-GGT gene was further confirmed by reannotation of the trnT-GGT region by ARAGORN v.1.2.38 [27] and tRNAscan-SE v2.0.7 [28]. The prediction of ARAGORN and/or tRNAscan-SE v2.0.7 was recorded for each species. The structural variations within trnT-GGT were analyzed by performing multiple alignment tool using clustalW [29] integrated into Geneious R8.1 and inspected manually at family, subfamily, and genus level. we analyzed codon usage of the five representative species to examine the effect of the pseudogenization of $\operatorname{trnT-GGU}$ on the sequences of protein-coding genes.

\section{Results}

\subsection{Analysis of trnT-GGU among species of Asteraceae}

We compare the trnT-GGU gene among 13 subfamilies of Asteraceae. The analyses revealed an insertion event (i.e TTTTT/TTTCT/TTCCT) occur at the $5^{\prime}$ acceptor stem in the species of subfamily Gymnarrhenoideae, Cichorioideae, Corymbioideae, and Asteroideae while lacking in the species of other subfamilies of Asteraceae (Figure 1). This insertion event was found linked to the pseudogenization of the trnT-GGU. The infernal score of the trnT-GGU gene was ranged from 65 to 49.4 in the species of those subfamilies, which lacked this insertion event (Table 1). The trnT-GGU gene was not annotated by the ARAGORN in the species of Gymnarrhenoideae, Cichorioideae, Corymbioideae, and Asteroideae after the aforementioned insertion. In contrast, tRNAScan-SE detected $\operatorname{trnT-GGU}$ gene as mismatch isotypes in 
Gymnarrhenoideae, with a low infernal score in Cichorioideae and Corymbioideae, and diverse types of results in the subfamily of Asteroideae (Table S2). The structure of trnT$G G U$ gene of the species of each subfamily showed that the mismatch/mismatches are present in the species of Gymnarrhenoideae, Cichorioideae, Corymbioideae, and Asteroideae mostly at $5^{\prime}$ acceptor stem and anticodon loop, whereas the species that lacked the aforementioned insertion has complete cloverleaf structure (Figure 2). This data showed the pseudogenization event might be widespread in the Asteraceae family.

\subsection{Analyses of trnT-GGU genes among the species of Carduoideae}

The analyses of 11 species from 11 different genera of Carduoideae showed that functional trnT-GGU gene with a high infernal score ranged from 55.7 to 57.1 (Table S3). Except for Atractylodes chinensis, the analyses of the other ten species showed the presence of anticodon CGU (Figure S1). We have also found an insertion (CTCAG) in the D-loop of Saussurea inversa, which a little slightly decreases the infernal score to 55.7. The structure analyses also support the presence of all functional parts of the gene in the species of Carduoideae (Figure 1e).

\subsection{Analyses of trnT-GGU genes among the species of Cichorioideae}

The analyses of 13 species from 13 genera of Cichorioideae revealed the pseudogenization of trnT-GGU gene in four species. The tRNAScan-SE predicted trnT-GGU in Hypochaeris radicata with mismatch isotypes of lysine along with truncated start and truncated end, Lactuca raddeana pseudogene (Figure S2). In Stebbinsia umbrella and Ixeris polycephala, the gene was not predicted due to deletion events. The structure of the species Hypochaeris radicata, and Lactuca raddeana showed certain mismatches at the acceptor stem and specific variations in the variable loop (Figure 3). In other species, the trnT-GGU gene was predicted with a low infernal score 31-34.6 (Table S4).

\subsection{Analyses of trnT-GGU genes among the species of Asteroideae}

We analyzed 97 species belonging to 78 genera and 13 tribes of the Asteroideae subfamily. The analyses revealed that the $\operatorname{trn} T-G G U$ gene exists as a pseudogene in the species of all the tribes (Table S4). This gene was not detected by ARAGORN and tRNAScan-SE in 61 species that were distributed in all the 13 tribes, pseudogene in 9 species, as mismatch isotypes of isoleucine and lysine in 16 species (Table S2). We also detected this gene with a low infernal score 22.6 in 12 species. However, the manual analyses of the structure revealed 
truncation at $5^{\prime}$ and $3^{\prime}$ end, which revealed that the gene might also be non-functional in these species. The structure of representative species is shown in figure 3 . The pseudogenization of the $\operatorname{trn} T-G G U$ gene occurs throughout the Asteroideae subfamily due to high mutational rate (substitutions and insertion-deletion) in all the functional parts of the genes. However, the highest mutations and degradation were recorded in the 5' acceptor arm, and $3^{\prime}$ arm (Figure S3). The pseudogenization was occurred in the species of Asteroideae irrespective of the habit, habitat, and native range (Table S1, Table S2).

\subsection{Analyses of Aldama, Artemisia, and Diplostephium species}

We analyzed 24 species of Artemisia, 21 species of Aldama, and 28 species of Diplostephium. The analyses showed the high similarities in the pseudogene of the $\operatorname{trnT-GGU}$ gene and showed fewer variations exist among the species of the same genus (Figure S4, Figure S5, Figure S6, Data file 1).

\subsection{Codon usage analyses}

The codon usage analyses of five representative species revealed high similarities in the codon usage for amino acid threonine in the species that have $\operatorname{trnT-GGU}$ as a pseudogene and in the species that contain a functional trnT-GGU gene (Table 2, Table S5). These findings

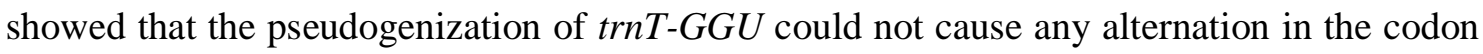
of protein-coding sequences.

\section{Discussion}

In the present study, the structure of the trnT-GGU gene was investigated in twelve subfamilies of Asteraceae. Our findings show the pseudogenization of $\operatorname{trn} T-G G U$ gene in the species of Gymnarrhenoideae, Cichorioideae, and Asteroideae irrespective of habit, habitat, and geographical distribution. Besides, pseudogenization of the $\operatorname{trnT-GGU}$ was reported in previous studies, including the Cryptomeria japonica D. Don. Of family Cupressaceae [30], Pelargonium x hortorum of family Geraniaceae [31]. Lee et al. [25] also reported $\operatorname{trnT-GGU}$ as a pseudogene in the two species of the tribe Gnaphalieae of Asteroideae (Asteraceae). However, they suggested that this pseudogenization might be limited to the species of tribe Gnaphalieae. Our study showed that due to insertion in the $5^{\prime}$ acceptor loop, the pseudogenization of the gene might occur which was extended into the three subfamilies of Asteraceae, including Gymnarrhenoideae, Cichorioideae, and Asteroideae and was not limited to the tribe Gnaphalieae. It reveals that the independent modifications have occurred 
during evolution in trnT-GGU gene as well as chloroplast genome of Asteraceae family. However, the similar mutation patterns were found in several subfamilies of Asteraceae. Previous studies showed that insertions-deletions generate substitutions [21,32-34] due to the recruitment of error-prone DNA polymerase [35,36]. Hence, the insertion may increase the

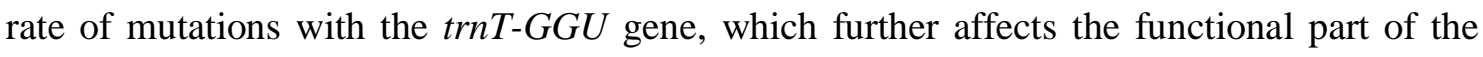
gene. Also, it was found that the variation of $\operatorname{trnT-GGU}$ gene in the chloroplast genome can be liked with genetic rearrangements [30,31]. Identification the variation and type of mutations can provide a new insight for better understanding of the function and regulatory mechanisms of $\operatorname{trnT} T-G G U$ gene in land plants.

The inversion in the chloroplast genome mostly did not cause pseudogenization of the genes [5]. However, in some lineages, the inversion was also found linked to pseudogenization [31]. Previously, the long inversion event $(22.8 \mathrm{~kb})$ was reported in the species of Asteraceae except for Barnadesioideae. The one endpoint of this inversion was between $\operatorname{trnS}-G C U$ and $\operatorname{trn} G-U C C$ genes, whereas the other endpoint was between trnE-UUC and trnT-GGU [37]. The pseudogenization of the trnT-GGU was not occurred in the species of Mutisioideae, Carduoideae, and Pertyoideae along with the species of Barnadesioideae. Hence, this revealed that the inversion event is not responsible for the pseudogenization of the $\operatorname{trnT-GGU}$ gene in the Asteraceae family. The loss of the trnT-GGU was not reflected in codon usage. This is an agreement with the previous report of Pelargonium $\mathrm{x}$ hortorum of family Geraniaceae [31]. Some mechanisms might be occurred to accommodate the loss of the trnT$G G U$ gene. Moreover, functional study of the gene can provide new insights into the loss and the mechanisms occur in these plant species.

The pseudogenization of the trnT-GGU gene was found in the species of diverse habit, habitat, and geographical distribution of the species (Table S1, Table S3). This showed the pseudogenation is not linked to convergent evolution and can provide insight into the evolution and phylogenetics of the family Asteraceae as suggested previously [25]. Moreover, the pseudogenization of the $\operatorname{trn} T-G G U$ also agreed with the previous phylogeny of the family Asteraceae $[1,4]$.

In conclusion, we report the pseudogenization of the trnT-GGU gene in the four subfamilies of Asteraceae after analyses of the high number of plant species, specifically of the subfamily Asteroideae. This will broaden the knowledge about the evolution of the chloroplast genome in the angiosperms. In addition, in the current study, diverse mutation events were observed 
into the trnT-GGU gene sequence that could be investigated in future studies related to functional genomics of chloroplast genome.

\section{Conflict of interest}

No conflict of interest exists.

\section{Data availability}

All the data are publicly available and mentioned in the manuscript. The analyses are included in the main manuscript or in the supplementary table. 


\section{References}

[1] J.R. Mandel, R.B. Dikow, C.M. Siniscalchi, R. Thapa, L.E. Watson, V.A. Funk, A fully resolved backbone phylogeny reveals numerous dispersals and explosive diversifications throughout the history of Asteraceae, Proc. Natl. Acad. Sci. U. S. A. 116 (2019) 14083-14088. doi:10.1073/pnas.1903871116.

[2] V.D. Barreda, L. Palazzesi, M.C. Tellería, E.B. Olivero, J.I. Raine, F. Forest, Early evolution of the angiosperm clade Asteraceae in the Cretaceous of Antarctica, Proc. Natl. Acad. Sci. U. S. A. 112 (2015) 10989-10994. doi:10.1073/pnas.1423653112.

[3] J.L. Panero, S.E. Freire, L. Ariza Espinar, B.S. Crozier, G.E. Barboza, J.J. Cantero, Resolution of deep nodes yields an improved backbone phylogeny and a new basal lineage to study early evolution of Asteraceae, Mol. Phylogenet. Evol. 80 (2014) 4353. doi:10.1016/j.ympev.2014.07.012.

[4] J.L. Panero, B.S. Crozier, Macroevolutionary dynamics in the early diversification of Asteraceae, Mol. Phylogenet. Evol. 99 (2016) 116-132. doi:10.1016/j.ympev.2016.03.007.

[5] H. Daniell, C.-S. Lin, M. Yu, W.-J. Chang, Chloroplast genomes: diversity, evolution, and applications in genetic engineering., Genome Biol. 17 (2016) 134.

doi:10.1186/s13059-016-1004-2.

[6] J.D. Palmer, Comparative organization of chloroplast genomes, Annu. Rev. Genet. 19 (1985) 325-354. doi:10.1146/annurev.ge.19.120185.001545.

[7] D.B. Neale, R.R. Sederoff, Paternal inheritance of chloroplast DNA and maternal inheritance of mitochondrial DNA in Loblolly pine, Theor. Appl. Genet. 77 (1989) 212-216. doi:10.1007/BF00266189.

[8] H. Daniell, Transgene containment by maternal inheritance: effective or elusive?, Proc. Natl. Acad. Sci. U. S. A. 104 (2007) 6879-6880. doi:10.1073/pnas.0702219104.

[9] I. Ahmed, P.J. Lockhart, E.M.G. Agoo, K.W. Naing, D. V. Nguyen, D.K. Medhi, P.J. Matthews, Evolutionary origins of taro ( Colocasia esculenta ) in Southeast Asia, Ecol. Evol. (2020) 1-14. doi:10.1002/ece3.6958.

[10] F. Mehmood, Abdullah, Z. Ubaid, I. Shahzadi, I. Ahmed, M.T. Waheed, P. Poczai, B. Mirza, Plastid genomics of Nicotiana (Solanaceae): insights into molecular evolution, 
positive selection and the origin of the maternal genome of Aztec tobacco (Nicotiana rustica), PeerJ. 8 (2020) e9552. doi:10.1101/2020.01.13.905158.

[11] C.L. Henriquez, Abdullah, I. Ahmed, M.M. Carlsen, A. Zuluaga, T.B. Croat, M.R. Mckain, Evolutionary dynamics of chloroplast genomes in subfamily Aroideae (Araceae), Genomics. 112 (2020) 2349-2360. doi:10.1016/j.ygeno.2020.01.006.

[12] C.L. Henriquez, Abdullah, I. Ahmed, M.M. Carlsen, A. Zuluaga, T.B. Croat, M.R. Mckain, Molecular evolution of chloroplast genomes in Monsteroideae (Araceae), Planta. 251 (2020) 72. doi:10.1007/s00425-020-03365-7.

[13] Abdullah, C.L. Henriquez, F. Mehmood, A. Hayat, A. Sammad, S. Waseem, M. Tahir, P.J. Matthews, T.B. Croat, P. Poczai, I. Ahmed, Chloroplast genome evolution in the Dracunculus clade (Aroideae , Araceae), Genomics. 113 (2021) 183-192. doi:10.1016/j.ygeno.2020.12.016.

[14] Abdullah, S. Waseem, B. Mirza, I. Ahmed, M.T. Waheed, Comparative analyses of chloroplast genomes of Theobroma cacao and Theobroma grandiflorum, Biologia (Bratisl). 75 (2020) 761-771. doi:10.2478/s11756-019-00388-8.

[15] I. Shahzadi, Abdullah, F. Mehmood, Z. Ali, I. Ahmed, B. Mirza, Chloroplast genome sequences of Artemisia maritima and Artemisia absinthium: Comparative analyses, mutational hotspots in genus Artemisia and phylogeny in family Asteraceae, Genomics. 112 (2020) 1454-1463. doi:10.1016/J.YGENO.2019.08.016.

[16] Abdullah, C.L. Henriquez, F. Mehmood, I. Shahzadi, Z. Ali, M.T. Waheed, T.B. Croat, P. Poczai, I. Ahmed, Comparison of chloroplast genomes among Species of Unisexual and Bisexual clades of the monocot family Araceae, Plants. 9 (2020) 737. doi:10.3390/plants9060737.

[17] F. Mehmood, Abdullah, Z. Ubaid, Y. Bao, P. Poczai, Comparative Plastomics of Ashwagandha ( Withania, Solanaceae) and Identification of Mutational Hotspots for Barcoding Medicinal Plants, Plants. 9 (2020) 752.

[18] F. Mehmood, Abdullah, I. Shahzadi, I. Ahmed, M.T. Waheed, B. Mirza, Characterization of Withania somnifera chloroplast genome and its comparison with other selected species of Solanaceae, Genomics. 112 (2020) 1522-1530. doi:10.1016/j.ygeno.2019.08.024. 
[19] Abdullah, I. Shahzadi, F. Mehmood, Z. Ali, M.S. Malik, S. Waseem, B. Mirza, I. Ahmed, M.T. Waheed, Comparative analyses of chloroplast genomes among three Firmiana species: Identification of mutational hotspots and phylogenetic relationship with other species of Malvaceae, Plant Gene. 19 (2019) 100199. doi:10.1016/J.PLGENE.2019.100199.

[20] P. Poczai, J. Hyvönen, Identification and characterization of plastid trnF (GAA) pseudogenes in four species of Solanum (Solanaceae), Biotechnol. Lett. 33 (2011) 2317-2323. doi:10.1007/s10529-011-0701-x.

[21] Abdullah, F. Mehmood, I. Shahzadi, S. Waseem, B. Mirza, I. Ahmed, M.T. Waheed, Chloroplast genome of Hibiscus rosa-sinensis (Malvaceae): Comparative analyses and identification of mutational hotspots, Genomics. 112 (2020) 581-591. doi:10.1016/j.ygeno.2019.04.010.

[22] A. Zuluaga, M. Llano, K. Cameron, Systematics, Biogeography, and Morphological Character Evolution of the Hemiepiphytic Subfamily Monsteroideae (Araceae), Ann. Missouri Bot. Gard. 104 (2019) 33-48. doi:10.3417/2018269.

[23] K. Vijverberg, K. Bachmann, Molecular evolution of a tandemly repeated trnF(GAA) gene in the chloroplast genomes of Microseris (Asteraceae) and the use of structural mutations in phylogenetic analyses, Mol. Biol. Evol. 16 (1999) 1329-1340. doi:10.1093/oxfordjournals.molbev.a026043.

[24] M.A. Koch, C. Dobeš, C. Kiefer, R. Schmickl, L. Klimeš, M.A. Lysak, Supernetwork Identifies Multiple Events of Plastid trnF(GAA) Pseudogene Evolution in the Brassicaceae, Mol. Biol. Evol. 24 (2007) 63-73. doi:10.1093/molbev/msl130.

[25] D.H. Lee, W.B. Cho, B.H. Choi, J.H. Lee, Characterization of two complete chloroplast genomes in the tribe gnaphalieae (Asteraceae): Gene loss or pseudogenization of trnT-GGU and implications for phylogenetic relationships, Korean J. Hortic. Sci. Technol. 35 (2017) 769-783. doi:10.12972/kjhst.20170081.

[26] M. Kearse, R. Moir, A. Wilson, S. Stones-Havas, M. Cheung, S. Sturrock, S. Buxton, A. Cooper, S. Markowitz, C. Duran, T. Thierer, B. Ashton, P. Meintjes, A. Drummond, Geneious Basic: An integrated and extendable desktop software platform for the organization and analysis of sequence data, Bioinformatics. (2012). doi:10.1093/bioinformatics/bts199. 
[27] D. Laslett, B. Canback, ARAGORN, a program to detect tRNA genes and tmRNA genes in nucleotide sequences, Nucleic Acids Res. 32 (2004) 11-16.

doi:10.1093/nar/gkh152.

[28] P.P. Chan, T.M. Lowe, tRNAscan-SE: Searching for tRNA genes in genomic sequences, in: Methods Mol. Biol., Humana Press Inc., 2019: pp. 1-14.

doi:10.1007/978-1-4939-9173-0_1.

[29] M.A. Larkin, G. Blackshields, N.P. Brown, R. Chenna, P.A. McGettigan, H.

McWilliam, F. Valentin, I.M. Wallace, A. Wilm, R. Lopez, Clustal W and Clustal X version 2.0, Bioinformatics. 23 (2007) 2947-2948.

[30] T. Hirao, A. Watanabe, M. Kurita, T. Kondo, K. Takata, Complete nucleotide sequence of the Cryptomeria japonica D. Don. chloroplast genome and comparative chloroplast genomics: Diversified genomic structure of coniferous species, BMC Plant Biol. 8 (2008). doi:10.1186/1471-2229-8-70.

[31] T.W. Chumley, J.D. Palmer, J.P. Mower, H.M. Fourcade, P.J. Calie, J.L. Boore, R.K. Jansen, The complete chloroplast genome sequence of Pelargonium $\times$ hortorum: Organization and evolution of the largest and most highly rearranged chloroplast genome of land plants, Mol. Biol. Evol. 23 (2006) 2175-2190.

doi:10.1093/molbev/ms1089.

[32] Abdullah, C.L. Henriquez, T.B. Croat, P. Poczai, I. Ahmed, Mutational dynamics of aroid chloroplast genomes II, Front. Genet. 11 (2021) 610838. doi:10.1093/gbe/evs110.

[33] Abdullah, F. Mehmood, I. Shahzadi, Z. Ali, M. Islam, M. Naeem, B. Mirza, P. Lockhart, I. Ahmed, M.T. Waheed, Correlations among oligonucleotide repeats, nucleotide substitutions and insertion-deletion mutations in chloroplast genomes of plant family Malvaceae, J. Syst. Evol. (2020). doi:10.1111/jse.12585.

[34] I. Ahmed, P.J. Biggs, P.J. Matthews, L.J. Collins, M.D. Hendy, P.J. Lockhart, Mutational dynamics of aroid chloroplast genomes, Genome Biol. Evol. 4 (2012) 1316-1323. doi:10.1093/gbe/evs110.

[35] D. Tian, Q. Wang, P. Zhang, H. Araki, S. Yang, M. Kreitman, T. Nagylaki, R. Hudson, J. Bergelson, J.-Q. Chen, Single-nucleotide mutation rate increases close to 
insertions/deletions in eukaryotes, Nature. 455 (2008) 105-108.

doi:10.1038/nature07175.

[36] L. Zhu, Q. Wang, P. Tang, H. Araki, D. Tian, Genomewide Association between Insertions/Deletions and the Nucleotide Diversity in Bacteria, Mol. Biol. Evol. 26 (2009) 2353-2361. doi:10.1093/molbev/msp144.

[37] K.J. Kim, K.S. Choi, R.K. Jansen, Two chloroplast DNA inversions originated simultaneously during the early evolution of the sunflower family (Asteraceae), Mol. Biol. Evol. 22 (2005) 1783-1792. doi:10.1093/molbev/msi174. 
Figure 1. Multiple alignment of trnT-GGU gene among the species of 13 subfamilies of Asteraceae. All functional part of the gene has been mentioned above the alignment. The insertion occurred in the acceptor arm is highlighted. The co-occurrence among the mutational events is also shown above and below the alignment.

Figure 2. The structure of $t r n T-G G U$ gene of species of all the subfamilies. One species was taken from each subfamily. The trnT-GGU gene of Barnadesia lehmannii was label to show the functional parts as representative of all the species. The perfect cloverleaf structure of trnT-GGU exist in the species of nine subfamilies. The insertion occurred in the species of four subfamilies which also arise to mismatches above the anticodon loop. These were highlighted with red box.

Figure 3. The structure of pseudo or of low infernal score trnT-GGU gene. The structure of gene from a-f show species of Asteroideae whereas the g-i represent species of Cichorioideae. (a) and (b) The gene is pseudo due to loss of acceptor stem. (c and d). The gene are predicted with low infernal score (22.6) only by tRNAscan-SE. However, the mismatch at $5^{\prime}$ and $3^{\prime}$ indicates this gene may be also non-functional. (e and f). The gene is predicted as mismatch isotypes for isoleucine. The clear insertion is visible in the acceptor stem which disturb the cloverleaf structure. (g) loss of acceptor stem occurs similar to a and b. (f) indicate pseudo gene. (h) indicate the gene with low infernal score of 34.6. All the species show the mismatch of c-c which form an extra loop like structure above the codon loop. * indicate loss of acceptor arm, ** indicate mismatch at $5^{\prime}$ and $3^{\prime},+$ indicate the missing in the acceptor arm due to insertion. 
bioRxiv preprint doi: https://doi.org/10.1101/2021.02 01.429200; this version posted February 2, 2021. The copyright holder for this preprint (which was not certified by peer review) is the author/funder, who has granted bioRxiv a license to display the preprint in perpetuity. It is made available under aCC-BY-NC-ND 4.0 International license.

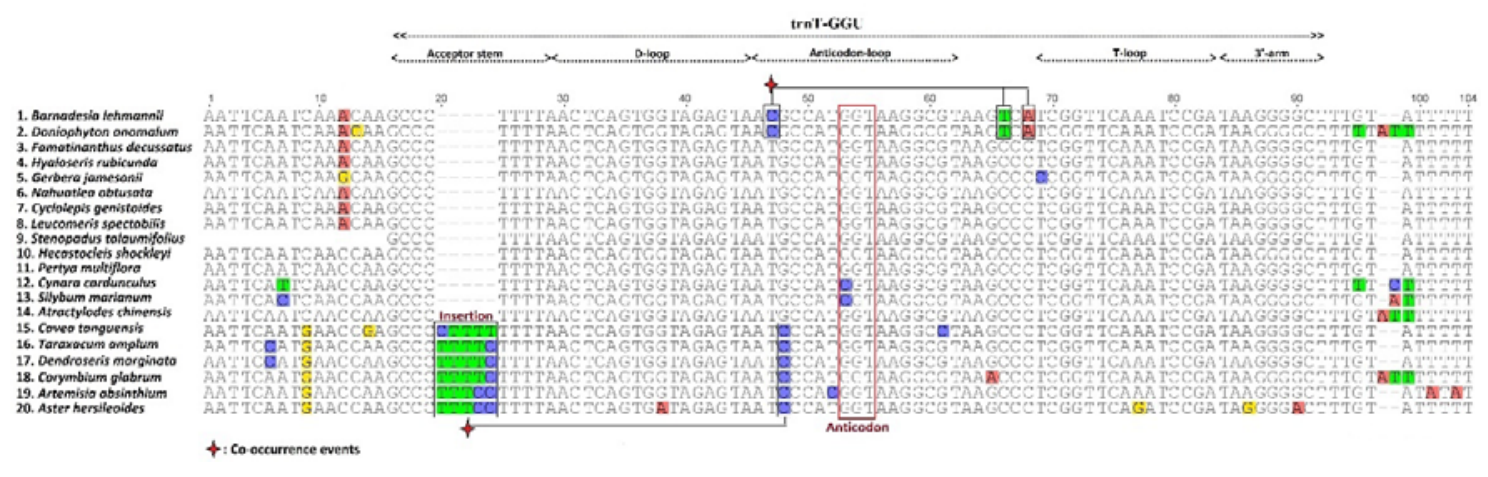


bioRxiv preprint doi: https://doi.org/10.1101/2021.02.01.429200; this version posted February 2, 2021. The copyright holder for this preprint (which was not certified by peer review) is the author/funder, who has granted bioRxiv a license to display the preprint in perpetuity. It is made available under aCC-BY-NC-ND 4.0 International license.
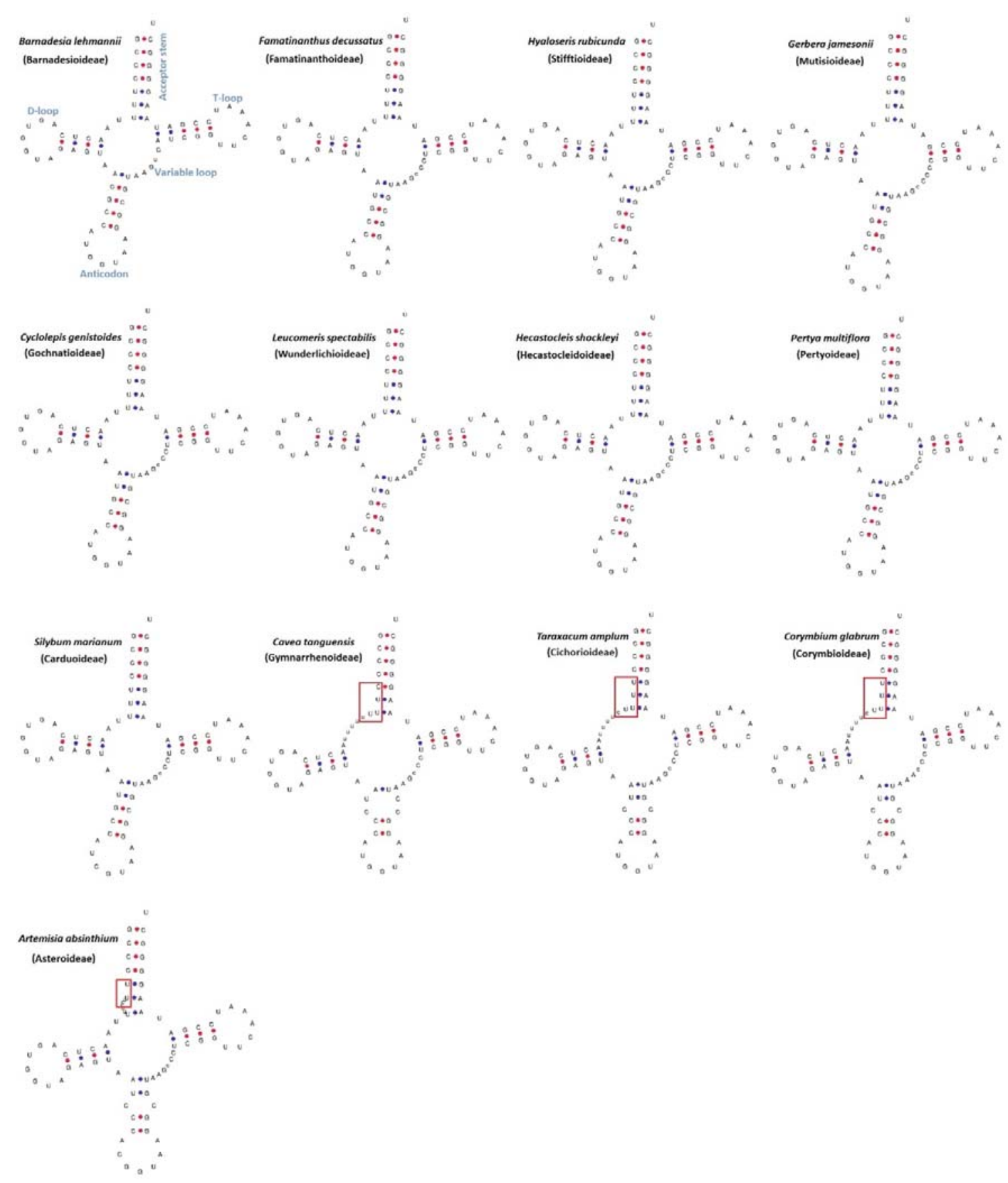
bioRxiv preprint doi: https://doi.org/10.1101/2021.02.01.429200; this version posted February 2, 2021. The copyright holder for this preprint (which was not certified by peer review) is the author/funder, who has granted bioRxiv a license to display the preprint in perpetuity. It is made available under aCC-BY-NC-ND 4.0 International license.

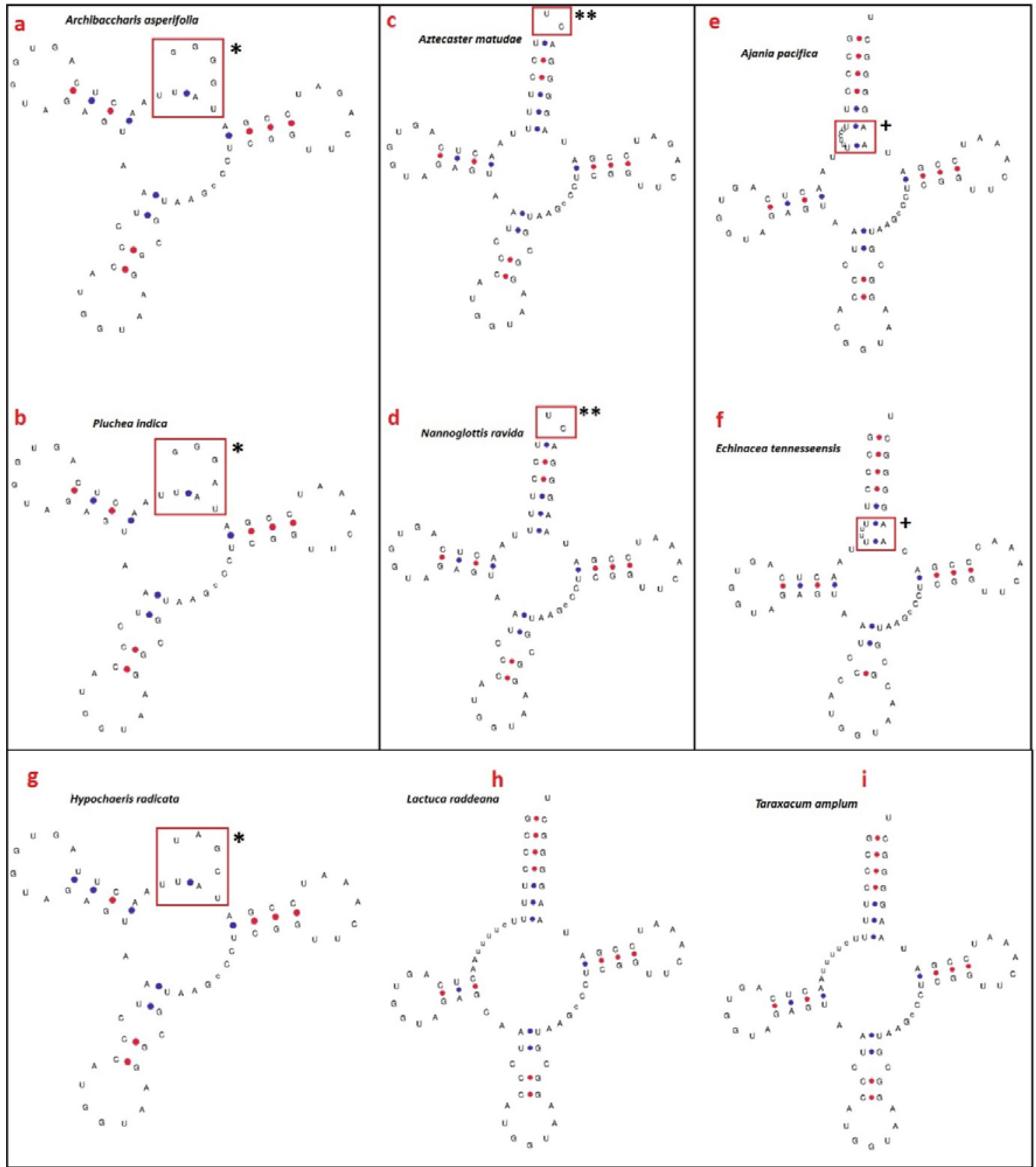


Table 1. Prediction of trnT-GGU genes in the representative species of 13 subfamilies

\begin{tabular}{|c|c|c|c|c|c|c|}
\hline S.No & Species & $\begin{array}{l}\text { tRNAScan } \\
\text { Prediction }\end{array}$ & $\begin{array}{c}\text { Infernal } \\
\text { score }\end{array}$ & Isotype & Anticodon & Subfamily \\
\hline 1 & Doniophyton anomalum & Thr & 65.8 & Thr & GGU & Barnadesioideae \\
\hline 2 & Barnadesia lehmannii & Thr & 65.8 & Thr & GGU & Barnadesioideae \\
\hline 3 & $\begin{array}{c}\text { Famatinanthus } \\
\text { decussatus }\end{array}$ & Thr & 57.1 & Thr & GGU & Famatinanthoideae \\
\hline 4 & Hyaloseris rubicunda & Thr & 57.1 & Thr & GGU & Stifftioideae \\
\hline 5 & Gerbera jamesonii & Thr & 49.4 & Thr & GGU & Mutisioideae \\
\hline 6 & Cyclolepis genistoides & Thr & 57.1 & Thr & GGU & Gochnatioideae \\
\hline 7 & Nahuatlea obtusata & Thr & 57.1 & Thr & GGU & Gochnatioideae \\
\hline 8 & Leucomeris spectabilis & Thr & 57.1 & Thr & GGU & Wunderlichioideae \\
\hline 9 & Stenopadus talaumifolius & Thr & 57 & Thr & GGU & Wunderlichioideae \\
\hline 10 & Hecastocleis shockleyi & Thr & 57.1 & Thr & GGU & Hecastocleidoideae \\
\hline 11 & Pertya multiflora & Thr & 57.1 & Thr & GGU & Pertyoideae \\
\hline 12 & Atractylodes chinensis & Thr & 57.1 & Thr & GGU & Carduoideae \\
\hline 13 & Cynara cardunculus & Thr & 57 & Thr & $\mathrm{CGU}$ & Carduoideae \\
\hline 14 & Silybum marianum & Thr & 57 & Thr & $\mathrm{CGU}$ & Carduoideae \\
\hline 15 & Cavea tanguensis & tRNA* & 32.7 & Ile 2 & GGU & Gymnarrhenoideae \\
\hline 16 & Dendroseris berteroana & Thr & 34.6 & Thr & GGU & Cichorioideae \\
\hline 17 & Taraxacum amplum & Thr & 34.6 & Thr & GGU & Cichorioideae \\
\hline 18 & Corymbium glabrum & Thr & 32.6 & Thr & GGU & Corymbioideae \\
\hline 19 & Artemisia ordosica & tRNA* & 27.8 & Ile2 & GGU & Asteroideae \\
\hline 20 & Aster hersileoides & Not detected & N/A & N/A & GGU & Asteroideae \\
\hline
\end{tabular}

* tRNAs with mismatch isotypes

Table 2. Codon usage comparison among the species with functional and non-functional trnT-GGU gene

\begin{tabular}{|c|c|c|c|c|r|r|}
\hline \multirow{2}{*}{ Codon } & \multirow{2}{*}{$\begin{array}{c}\text { Amio } \\
\text { acid }\end{array}$} & \multicolumn{6}{|c|}{$\begin{array}{l}\text { Number of codons } \\
\text { ordosica }\end{array}$} & $\begin{array}{l}\text { Aster } \\
\text { hersileoides }\end{array}$ & $\begin{array}{l}\text { Symphyotrichum } \\
\text { subulatum }\end{array}$ & $\begin{array}{l}\text { Helianthus } \\
\text { annuus }\end{array}$ & $\begin{array}{l}\text { Barnadesia } \\
\text { lehmannii }\end{array}$ \\
\hline ACA & Thr & 412 & 405 & 399 & 400 & 400 \\
\hline ACC & Thr & 241 & 240 & 237 & 236 & 247 \\
\hline ACG & Thr & 124 & 141 & 142 & 135 & 138 \\
\hline ACT & Thr & 527 & 530 & 536 & 519 & 537 \\
\hline
\end{tabular}

